\title{
Assessment of Commercially Available Computerized Neurocognitive Testing in the Adolescent Concussed Athlete: A Retrospective Analysis
}

Brock Hardin, MD', Andrew Gilliland, MD', Thomas Belmaggio, MS, ATC', Charles Giangarra, MD', John R. Castillo, BS', Mark Timmons, PhD, ATC'

\section{ABSTRACT}

Clinicians frequently use computer-based neurocognitive assessments to aid in the diagnosis and man-agement of Sport-Related Concussion (SRC). With practitioners using varied Neuro-Cognitive Assess-ment Tools (NCAT), questions arise concerning differences among NCAT and how these differences may affect patient care. The purpose of the current study is to offer a comparative analysis of two widely accepted, commercially available computer-based neurocognitive testing modalities in the adolescent con-cussed athlete.
Author affiliations are listed at the end of this article.

Correspondence to: Mark Timmons, PhD, ATC Marshall Unviersity Joan C. Edwards School of Medicine timmonsm@marshall.edu

\section{KEYWORDS}

C3 Logix, ImPACT, Neurocognitive Assessment Testing, Sports-Related Concussion,

Return to Play

\section{INTRODUCTION}

The incidence of sport-related concussion (SRC) is a growing problem in the field of sports medicine. Zhang et al. ${ }^{1}$ demonstrated that the incidence of concussion in the US population is increasing, particularly in adolescent populations. The assessment and management of patients following a head injury can be difficult. There is ongoing research within the healthcare community for the optimal assessment and treatment protocol for these patients. Standard practices are evolving, and consensus documents attempt to suggest "best evidence" standards for the patient with SRC., ${ }^{2,3}$ Neurocognitive Assessment Testing (NCAT) is a validated objective diagnostic and prognostication tool. ${ }^{4}$ NCATs are used by healthcare providers in the clinic setting, as well as in athletic training rooms to assist the management of patients with concussion.

Computer-based testing is now commonly used in tandem with symptom scoring tools. 5 There are several commercially available NCAT products competing to capture shares of a burgeoning market. It is unclear which product is superior with an increasing number of NCATs available. There is insufficient evidence to establish benefit in using a specific NCAT product for the evaluation, management, or monitoring of patients with SRC. There appears to be no clear clinical practice standard for the use of NCATs outside of manufacturer guidelines. Practitioner preference and familiarity with an NCAT product leads to varying practices among healthcare providers.

NCATs offer several potential advantages over the archetypal paper neuropsychological tests, such as the Sport Concussion Assessment Tool (SCAT5), in the assessment of patients with SRC. The ability of NCAT's to diagnose, rule out, and monitor recovery from SRC is unclear as many external factors can influence the utility of NCATs. There is a lack of evidence to support the reliability, validity, or clinical utility of NCATs. The literature provides some support for the convergent validity of NCAT tests.6,7 The results of reliability studies are variable. 4 It has been shown that a 
score on an NCAT which indicates a good cognitive function is useful in identifying patients without concussion.8 However, the utility of an NCAT to rule out SRC is not well documented.9,10 There have been concerns raised about the validity of these tests outside the acute 48-hour time frame. It is generally agreed that NCATs should not be used in isolation to diagnose or to guide the management of SRC until there is more evidence supporting the reliability and validity of NCATs.

The purpose of this retrospective cohort study is to characterize important translational metrics in athletic patients affected by sport-related concussion. The investigation determined differences in the time to the initiation of a return to play protocol (IRTP), the time to return to play (RTP), the level of impairment and concussion symptoms at the initial assessment, at the IRTP and the RTP when the clinician was assisted by either the ImPACT $^{\circledR}$ or $\mathrm{C}$ Logix $^{\circledR}$ NCAT. The investigation tested two hypotheses. First, there would be a difference between the C3 Logix ${ }^{\circledR}$ vs. ImPACT ${ }^{\circledR}$ scoring in the IRPT and RTP, and second, isolated NCAT symptom scores alone are not indicative of RTP; the majority of this decision relies on other factors to be managed by clinician care.

\section{METHODS}

Patients in the current study were entered into the study from patients treated by two sports medicine clinics from fall 2015 to spring 2017. A search of the practices' electronic medical record (Allscripts EHR System, Allscripts Healthcare Solutions, Chicago, IL, and EPIC Electronic Medical record) was conducted during November 2017. All records were identified with the diagnosis code of S06.0X0 (concussion without loss of consciousness), S06.0X9 (concussion with loss of consciousness of unspecified duration), or S06.0X1 (concussion with loss of consciousness less than 30 minutes). Patient records were included if the patient was 13-18 years of age, participated in an interscholastic athletic program, and had a diagnosis of an SRC. The project was approved by the University Internal Review Board (IRBNet ID\# 6273213). The current study was a retrospective review of records involving procedures that are considered the standard of care. No patient informed consent was required.

\section{PROCEDURE}

Patients included in this study participated in the Sports Medicine Clinic's baseline concussion testing program. Upon presentation, the treating physician performed a post-injury neurocognitive assessment and then managed the patient as is customary and usual to their practice. All patients were evaluated on their initial visit to the clinic with either the IMPACT $^{\circledR}$ or C3Logix ${ }^{\circledR}$ NCAT modality, depending on the preference of the treating physician. ImPACT ${ }^{\circledR}$ computerized testing is a widely accepted, wellmarketed platform. There is a growing amount of research supporting its utilization in an athletic medical setting. $11 \mathrm{ImPACT}^{\circledR}$ testing includes tests of verbal and visual memory, reaction time, symptom score, visual-motor speed, and impulse control. C3Logix ${ }^{\circledast}$ is a different platform developed by the Cleveland Clinic, has similar testing batteries, but also includes other metrics such as a vestibular baseline component.12 Repeat testing occurred as deemed necessary by the treating physician until it was determined that the patient was able to begin the return to play protocol.

\section{DATA ENTRY}

The records for all identified patients were reviewed by a single research assistant in order to identify the study's test variables. Patient identifying information was not extracted from the patient records. A second investigator reviewed the extracted data for accuracy before data analysis. The investigator identified the patient demographic information, the initial date of injury, date of the initial evaluation, the date of subsequent evaluations, the results of all clinical tests, NCAT results, date of the initiation of the return to play protocol, and the date of return to play. All data were entered into a spreadsheet and then imported into SPSS for analysis.

\section{STATISTICAL ANALYSIS}

Means and standard deviations for all study variables were calculated. All dependent variables followed 
a normal distribution determined by the ShapiroWilk test. The variance of dependent variables differed between groups, so Welch's t-tests were performed to determine between-group differences in symptom severity, duration of symptoms, time to the initiation of the return to play protocol, and time to return to play. Correlation analysis was used to test the relationship between symptom severity, the time to return to play protocol, and the time to return to play. Correlation coefficients $r=0-0.25$ were considered of no correlation, $r=0.26-0.50$ weak correlation, 0.51 - 0.75 moderate correlation, and $r>0.75$ strong correlation. Multiple regression analysis was used to determine if the initial symptom score of reaction time predicted either the time to initiation of the return to play protocol or the time to return to play. Statistical analysis was performed with SPSS 24.0 (SPSS Chicago, IL), and statistical significance was determined at $p<0.05$.

\section{RESULTS}

Two hundred twenty-two records (222) were entered into the study; five (5) records were missing either the date of injury or the date of the initial evaluation and were excluded from the investigation. Two hundred seventeen (217) records were entered into the analysis. One hundred ninety-two (192) records were evaluated using the $\mathrm{ImPACT}^{\circledR}$ testing, and twenty-five (25) were evaluated using the C3 Logix ${ }^{\circledast}$ system at the time of the initial evaluation. No patients included in the study received more than one recorded concussion during the data collection period. The mean time between the date of injury (DOI) and the initial evaluation (IEVAL), initiating the return to play protocol (IRPT) and the patients' return to play (RPT) can be found in Table 1. There was not a difference $(t=-1.226, P=0.22)$ in the time between

\begin{tabular}{|l|l|l|l|l|l|}
\hline & Mean & C3 Logix $®$ & ImPACT & T value & P-value \\
\hline IEVAL & $5.2 \pm 4.2$ & $6.2 \pm 4.3(\mathrm{n}=25)$ & $5.1 \pm 4.3(\mathrm{n}=192)$ & -1.229 & 0.228 \\
\hline IRTP & $15.1 \pm 18.4$ & $12.1 \pm 4.9(\mathrm{n}=19)$ & $15.6 \pm 19.8(\mathrm{n}=110)$ & 1.544 & 0.125 \\
\hline RTP & $21.5 \pm 24.9$ & $18.5 \pm 0.5(\mathrm{n}=2)$ & $21.7 \pm 4.5(\mathrm{n}=32)$ & $0 . .697$ & 0.491 \\
\hline
\end{tabular}

TABLE 1. Time (days) between the injury and the initial evaluation (IEVAL), the initiation of the return to play protocol (IRTP), and return to play (RTP), mean time \pm standard deviation.

\begin{tabular}{|l|l|l|l|l|l|}
\hline & Mean & C3 Logix & ImPACT $®$ & T value & P-value \\
\hline IEVAL & $23.9 \pm 22.3$ & $31.5 \pm 27.0(\mathrm{n}=12)$ & $23.2 \pm 21.9(\mathrm{n}=132)$ & -1.033 & 0.321 \\
\hline IRTP & $6.1 \pm 13.3$ & $6.8 \pm 18.7(\mathrm{n}=18)$ & $5.9 \pm 12.5(\mathrm{n}=137)$ & -0.202 & 0.842 \\
\hline RTP & $2.2 \pm 6.1$ & $0.750 \pm 0.018(\mathrm{n}=11)$ & $2.4 \pm 6.5(\mathrm{n}=144)$ & 1.192 & 0.239 \\
\hline
\end{tabular}

TABLE 2. Symptom score at the initial evaluation (IEVAL), the initiation of the return to play protocol (IRTP) and return to play (RTP), mean \pm standard deviation (mean \pm standard deviation) by each device, and the mean of the two devices.

\begin{tabular}{|l|l|l|l|l|l|}
\hline & Mean & C3 Logix & ImPACT & T value & P-value \\
\hline IEVAL & $0.743 \pm 0.016$ & $0.652 \pm 0.081(\mathrm{n}=11)$ & $0.750 \pm 0.214(\mathrm{n}=144)$ & 3.243 & 0.004 \\
\hline IRTP & $0.638 \pm 0.11$ & $0.623 \pm 0.115(\mathrm{n}=18)$ & $0.639 \pm 0.112(\mathrm{n}=132)$ & 0.566 & 0.577 \\
\hline RTP & $0.619 \pm 0.11$ & $0.593 \pm 0.123(\mathrm{n}=12)$ & $0.623 \pm 0.106(\mathrm{n}=83)$ & 0.802 & 0.436 \\
\hline
\end{tabular}

TABLE 3. Reaction time (seconds) at the initial evaluation (IEVAL), the initiation of the return to play protocol (IRTP) and return to play (RTP), mean \pm standard deviation (mean \pm standard deviation) by each device, and the mean of the two devices. 
the DOI and the IEVAL, IRTP or the RTP between patients evaluated using C3 Logix $^{\circledR}$ and ImPACT ${ }^{\circledR}$. The mean symptom score (Table 2 ) across both devices decreased during the time between IEVAL and RTP. Reaction time decreased between IEVAL and RTP (Table 3 ). The mean reaction time across both devices at the IEVAL was $0.743 \pm 0.016 \mathrm{sec}(n=155)$. The mean reaction time across both devices at the RTP was $0.619 \pm 0.11 \mathrm{sec}(n=95)$.

Weak to moderate correlations (Table 4) were found between symptom scores, reaction time, IRTP, and RTP. The time between the DOI and IEVAL did not correlate with symptom scores or reaction time. The strongest correlation $(r=0.551, P=0.01)$ was found between the time to IRTP and RTP. The symptom score at the IRTP had a weak correlation $(r=0.464$, $\mathrm{P}<0.001$ ) to the IEVAL symptom score. The reaction time and the symptom score at the IEVAL had a moderate correlation $(r=0.353, P<0.001)$. Multiple regression analysis revealed that the symptom score at the IEVAL predicted the IRPT $(r 2=0.214, \mathrm{P}$ $<0.001)$, reaction time factor but did not affect the regression model $(P=0.959)$ while the regression equation did not predict the RTP $(r 2=0.010, P=$ 0.91).

\section{DISCUSSION}

The purpose of the current study was to compare two popular and commercially used neurocognitive testing modalities in adolescent concussed athletes. Graham et $\mathrm{al}^{8}$ advocate for increased reliance on subjective means for the clinician to employ in their diagnoses and management of concussion injuries. Higgins et $\mathrm{al}^{13}$ and Simon et ${ }^{14}$ al identified ImPACT ${ }^{\circledR}$ and C3 Logix ${ }^{\circledR}$ as a valid means for testing symptom scores, respectively. Our results indicate the NCATs tested (C3 Logix $^{\circledR}$ and ImPACT ${ }^{\circledR}$ ) did not show a difference in their prediction of IRPT based upon symptom score alone. Two hundred seventeen (217) records were entered into the study for analysis, with one hundred ninety-two (192) evaluated using ImPACT $^{\circledR}$ testing, and twenty-five (25) using C3 Logix ${ }^{\circledR}$ testing. The two groups had a similar level of concussion symptoms at the initial evaluation. The mean time between IEVAL and initiation if IRTP protocol was $15 \pm 18$ days. The considerable deviations to this value are likely attributed to

\begin{tabular}{|c|c|c|c|c|c|c|}
\hline & $\begin{array}{l}\text { Time to } \\
\text { IRPT }\end{array}$ & $\begin{array}{l}\text { Time to } \\
\text { RTP }\end{array}$ & $\begin{array}{l}\text { Symptom } \\
\text { Score IE- } \\
\text { VAL }\end{array}$ & $\begin{array}{l}\text { Symptom } \\
\text { Score RTP }\end{array}$ & $\begin{array}{l}\text { Reaction } \\
\text { Time Ini- } \\
\text { tial }\end{array}$ & $\begin{array}{l}\text { Reaction } \\
\text { Time RTP }\end{array}$ \\
\hline Time to IRPT & & $\begin{array}{l}.551, \\
\mathrm{P}=0.010\end{array}$ & $\begin{array}{l}.464, \\
\mathrm{P}<0.001\end{array}$ & $\begin{array}{l}.142 \\
\mathrm{P}=293\end{array}$ & $\begin{array}{l}.093 \\
\mathrm{P}=397\end{array}$ & $\begin{array}{l}.001, \\
\mathrm{P}=0.997\end{array}$ \\
\hline Time to RTP & & & $\begin{array}{l}.100 \\
\mathrm{P}=0.667\end{array}$ & $\begin{array}{l}.532, \\
\mathrm{P}=0.092\end{array}$ & $\begin{array}{l}.055 \\
\mathrm{P}=0.802\end{array}$ & $\begin{array}{l}-.019 \\
\mathrm{P}=0.956\end{array}$ \\
\hline $\begin{array}{l}\text { Symptom } \\
\text { Score Initial }\end{array}$ & & & & $\begin{array}{l}.196 \\
\mathrm{P}=0.140\end{array}$ & $\begin{array}{l}.353 \\
\mathrm{P}<0.001\end{array}$ & $\begin{array}{l}.019 \\
\mathrm{P}=0.891\end{array}$ \\
\hline $\begin{array}{l}\text { Symptom } \\
\text { Score RTP }\end{array}$ & & & & & $\begin{array}{l}.028 \\
\mathrm{P}=821\end{array}$ & $\begin{array}{l}-.020 \\
\mathrm{P}=0.845\end{array}$ \\
\hline $\begin{array}{l}\text { Reaction Time } \\
\text { IEVAL }\end{array}$ & & & & & & $\begin{array}{l}.044, \\
\mathrm{P}=0.724\end{array}$ \\
\hline $\begin{array}{l}\text { Reaction Time } \\
\text { RTP }\end{array}$ & & & & & & \\
\hline
\end{tabular}

Table 4. Correlation coefficients, initiate the return to play protocol (IRPT), return to play (RPT), initial evaluation (IE-VAL). 
the variability in the level of symptom severity and clinician care based upon other subjective or objective criteria, such as individual medical history or presenting symptoms not captured by tested NCAT criteria. There was not a difference in the time between the DOI and RTP between patients evaluated with C3 Logix $^{\circledast}$ (12.1 \pm 4.9 days) and ImPACT $^{\oplus}(15.6 \pm 19.8$ days). This suggests there is not a major difference in the predictive nature of the NCAT testing modalities studied, which is counter to the hypothesis at the onset of the study.

Individuals that had an IRTP greater-than 12 days tended to have a greater symptom score at presentation, and individuals that had an IRTP lessthan 12 days tended to have a lesser symptom score at presentation with C3 Logix ${ }^{\circledR}$ testing. Individuals that had an IRTP greater-than 15 days tended to have a greater symptom score at presentation, and individuals that had an IRTP less-than 15 days tended to have a lesser symptom score at presentation with $\mathrm{ImPACT}^{\oplus}$ testing. Regression analysis revealed that the symptom score at the IEVAL predicted the IRTP $(r 2=0.21, p<0.001)$ but not the RTP $(r 2=0.01, p$ $=0.91)$. This is consistent with known research that symptom score is indicative of IRTP. ${ }^{15}$ However, the low R2 value indicates that still most of the influence comes from other factors that are involved in the managing and rehabilitation for concussion injuries.

Mean symptom scores decreased during the time between IEVAL and RTP for both C3 Logix ${ }^{\circledR}$ and ImPACT ${ }^{\oplus}$ testing. There was no difference observed in the symptom scores at IEVAL between C3 Logix ${ }^{\circledR}$ and ImPACT ${ }^{\circledast}$. For patients participating in the study, neither data set was more severely injured based upon presenting symptom score, and the pathology of injury recovery progressed equally for both testing modalities. Patients in the study followed a standardized recovery protocol derived from the Berlin consensus guidelines. ${ }^{2}$

The results of the current study must be reviewed in light of the following limitations. The study was limited by the disproportionate number tested using the two NCAT testing modalities, with a larger patient population tested with $\operatorname{ImPACT}^{\oplus}(n=192)$ than C3 Logix ${ }^{\circledast}(n=25)$. There was not a statistical difference in the symptom scores between the two devices. Due to the disproportioned sample sizes, the current study did not attempt to establish equivalence between the measures made using the two devices. The purpose of the investigation was to explore if differences between the two devices could be found not if the two devices are equivalent. The range of the scores was the same between the two devices. Five records were excluded due to incomplete data reducing the sample size to 217. Patients were evaluated and managed by five physicians with varying training. However, all were experienced in the treatment of concussed athletes. The largest number of patients were managed by a single investigator. The finding of long reaction time at IEVAL for patients evaluated with ImPACT ${ }^{\circledR}$ must be interpreted with caution. The two tests do not measure reaction time using the same criteria and this is likely a spurious finding. All of the clinicians managed their patients using the best available clinical evidence. Lastly, there was no attempt to control for concussion history or other comorbidities. It is likely concussion history and comorbidities occurred at equal rates in patients tested with both NCATs.

Zhang, et al ${ }^{1}$ identified a rise in the incidence of concussion in the adolescent athletic population; the increase in the number of concussive injuries calls for an increase in the understanding of how to diagnose and manage these patients. Further research connecting the increasingly widespread use of NCATs in both the office and sideline environment may be beneficial to identify a potential correlation between these two values. Our data agree with previous literature on the application of NCATs in the diagnosis and management of concussion injuries.

The relatively large deviation in IRTP and RTP for both testing devices is most likely attributed to the individualized patient care provided by clinicians, which concurs with Johnson et al.5 and the suggestion that NCATs can provide clinicians a valid objective means to provide direct care to treat targeted deficiencies in neurocognitive function, such as balance or visual acuity insufficiencies. Dessy et $\mathrm{al}^{11}$ identified that no single test is sufficient for the stand-alone diagnoses of sports-related concussions. Arrieux et $\mathrm{al}^{4}$ have identified there are still questions to the use of NCATs for an accurate diagnosis of concussion injuries. Our results expand upon these issues, indicating that two of the most 
common NCAT yield similar results; however, the largest influence remains in the tailored patient care provided by the clinician.

Research suggests as high as fifty-percent of concussions may go unreported ${ }^{16}$, which may pose challenges for healthcare providers. Further research and advocacy on the increasing use of NCATs may propose an actionable solution to decrease the number of unreported concussions. Additional research into the development of a specialized protocol for NCAT derived symptom scores, utilizing a variety of modalities, may be beneficial in the treatment of future patients. This may serve to provide screening tools that can assist the clinical judgment of healthcare providers. There may be circumstances that clinicians determine to remove an athlete from play and enter concussion protocol based upon other subjective or objective criteria when NCAT scores fall below the concussion threshold. Further research into the prevalence of this scenario may prove beneficial to understand the role NCATs play in the initial diagnoses of SRC injuries and their function as a means to measure recovery from the injury and provide recommendations for the return to play.

The current study focused on the quality and comparison of two widely used NCAT devices in their application to the concussed athlete as a means to predict a return to play. It did not focus on major differences between the two testing protocols and procedures directed by the respective companies. Our results indicated that NCATs provide a minority of predictive means for athlete return to play from the initial injury and showed two of the most widely used devices did not display a major difference in this area. Presenting symptom score alone was not sufficient to predict IRTP; however, it remained the most weighted testing measure across testing devices. The results of this study concur with established literature that the most important aspect to the individualized care of the concussed athlete remains the expertise and patient knowledge possessed by the managing clinician.

\section{AUTHOR AFFILIATIONS}

1. Marshall University Joan C. Edwards School of Medicine, Huntington, West Virginia

\section{REFERENCES}

1. Zhang AL, Sing DC, Rugg CM, Feeley BT, Senter $C$. The rise of concussions in the adolescent population. Orthop J Sports Med. 2016;4(8):2325967116662458.

2. Harmon KG, Clugston JR, Dec K, et al. American Medical Society for Sports Medicine Position statement on concussion in sport. Clin J Sport Med. 2019;29(2):87-100.

3. Herring SA, Cantu RC, Guskiewicz KM, et al. Concussion (mild traumatic brain injury) and the team physician: a consensus statement--2011 update. Med Sci Sports Exerc. 2011;43(12):24122422.

4. Arrieux JP, Cole WR, Ahrens AP. A review of the validity of computerized neurocognitive assessment tools in mild traumatic brain injury assessment. Concussion. 2017;2(1):CNC31.

5. Johnson EW, Kegel NE, Collins MW. Neuropsychological assessment of sport-related concussion. Clin Sports Med. 2011;30(1):73-88, viii-ix.

6. Farnsworth JL, Dargo L, Ragan BG, Kang M. Reliability of computerized neurocognitive tests for concussion assessment: A Meta-Analysis. J Athl Train. 2017;52(9):826-833.

7. Nakayama Y, Covassin T, Schatz P, Nogle S, Kovan J. Examination of the test-retest reliability of a computerized neurocognitive test battery. Am J Sports Med. 2014;42(8):2000-2005.

8. Graham R, Rivara FP, Ford MA, Spicer CM. Concussion recognition, diagnosis, and acute management. In: Graham R, Rivara FP, Ford MA, Spicer CM, eds. Sports-related concussions in youth: improving the science, changing the culture. Washington (DC)2014.

9. Alsalaheen B, Stockdale K, Pechumer D, Broglio SP. Measurement error in the immediate postconcussion assessment and cognitive testing (ImPACT): Systematic Review. J Head 
Trauma Rehabil. 2016;31(4):242-251.

10. Alsalaheen B, Stockdale K, Pechumer D, Broglio SP, Marchetti GF. A comparative meta-analysis of the effects of eoncussion on a computerized neurocognitive test and self-reported symptoms. J AthI Train. 2017;52(9):834-846.

11. Dessy AM, Yuk FJ, Maniya AY, et al. Review of assessment scales for diagnosing and monitoring sports-related concussion. Cureus. 2017;9(12):e1922.

12. Borges A, Raab S, Lininger M. A comprehensive instrument for evaluating mild traumatic brain injury (Mtbi)/concussion in independent adults: a pilot study. Int J Sports Phys Ther. 2017;12(3):381389.

13. Higgins KL, Caze T, Maerlender A. Validity and reliability of baseline testing in a standardized environment. Arch Clin Neuropsychol. 2018;33(4):437-443.

14. Simon M, Maerlender A, Metzger K, Decoster L, Hollingworth A, Valovich McLeod T. Reliability and concurrent validity of select C3 Logix test components. Dev Neuropsychol. 2017;42(78):446-459.

15. Merritt VC, Rabinowitz AR, Arnett PA. Injuryrelated predictors of symptom severity following sports-related concussion. J Clin Exp Neuropsychol. 2015;37(3):265-275.

16. McCrea M, Hammeke T, Olsen G, Leo P, Guskiewicz K. Unreported concussion in high school football players: implications for prevention. Clin J Sport Med. 2004;14(1):13-17. 